УДК 517.55, 517.923, 514.74

\title{
Decomposition of Functions of Finite Analytical Complexity
}

\author{
Valery K. Beloshapka* \\ Faculty of Mechanics and Mathematics \\ Moscow State University \\ Vorob'evy Gory, Moscow, 119991
}

Russia

Received 07.07.2018, received in revised form 01.08.2018, accepted 06.10.2018

\begin{abstract}
Functions of two variables can be obtained from functions of one variable by substitutions and additions. Each composition scheme corresponds to a class of functions of two variables, such that they can be represented as a composition with this scheme. It was shown that each class consists of analytical solutions of a certain system of differential polynomials (equations of the class). The paper describes an algorithm for constructing a system of equations of the scheme and for obtaining function representation in the form of a composition with this scheme.
\end{abstract}

Keywords: decomposition scheme, representation of the functions.

DOI: 10.17516/1997-1397-2018-11-6-680-685.

Let $Z$ be an analytical function of two variables and $(z(x, y), D)$ be an element of $Z$. It means that function $z(x, y)$ is holomorphic in $D$. Here $D$ is the 2-dimensional domain and $Z$ is the result of the analytic continuation of this element. Let us assume that analytical complexity of $Z$ is equal to one. This means that in $D$ there ares a point $\left(x_{0}, y_{0}\right)$ with the neighbourhood $V=\left\{\left|x-x_{0}\right|<\delta,\left|y-y_{0}\right|<\delta\right\}$ contained in $D$, a non-constant function $a(x)$ which is holomorphic in $\left\{\left|x-x_{0}\right|<\delta\right\}$, a non-constant function $b(y)$ which is holomorphic in $\left\{\left|y-y_{0}\right|<\delta\right\}$, a nonconstant function $c(t)$ which is holomorphic in $\left\{\left|t-\left(a\left(x_{0}\right)+b\left(y_{0}\right)\right)\right|<\varepsilon\right\}$ such that in $V$ there is a representation

$$
z(x, y)=c(a(x)+b(y))
$$

Throughout the paper we distinguish an analytical function $z$ which we plan to represent as a composition of a certain type and the composition $S$ itself.

A composition of a fixed form consisting of functions of one variable and addition that contains indeterminate and independent functions is thecomposition scheme.

To transform the scheme into a function of two variables one should define a set of germs of holomorphic functions of one variable.

In our example, the set of undefined functional variables is $(a, b, c)$. If instead of undefined variables $(a, b, c)$ we substitute germs of specific holomorphic functions $(A, B, C)$ then we obtain the germ of specific holomorphic function of two variables $S(A, B, C)(x, y)$ (or we say that this germ is undefined). In our example, we have $S(A, B, C)(x, y)=c(a(x)+b(y))$. In this approach, the question of representability of a germ of function $z(x, y)$ as a composition with this scheme $s$ can be formulated as follows.

Are there germs of functions of one variable $(A, B, C)$ such that $z(x, y)=S(A, B, C)(x, y)$ ?

Lemma 1. Let us assume that functions $\varphi(x, y)$ and $z(x, y)$ are defined in the neighbourhood of a point $p=\left(x_{0}, y_{0}\right)$, and they are holomorphic in this neighbourhood. Let us also assume that $p$ is not a critical point of $\varphi$, i.e., a partial derivative of $\varphi$ is not equal to zero at this point. Then function $z$ can be represented in the neighbourhood of $p$ as $z(x, y)=c(\varphi(x, y))$ if and only if

$$
\varphi_{y}^{\prime} z_{x}^{\prime}-\varphi_{x}^{\prime} z_{y}^{\prime}=0
$$

*valery@beloshapka.ru

(c) Siberian Federal University. All rights reserved 
in the neighbourhood of $p$.

Proof: Let us apply the implicit function theorem. Namely,we can replace coordinates $(x, y)$ with coordinates $(x, t)$ or $(t, y)$ in the neighbourhood of $p$, where $t=a(x)+b(y)$. Then it follows from (2) that $z$ is a function of only one variable $t$.

Corollary 2. If $z$ and $\varphi$ are holomorphic functions in $D$ then representation of function $z$ as $S(c, b, a)(x, y)=c(a(x)++b(y))$ can be extanded along all paths in $D$ that do not go through singular level lines of $\varphi$ (the discriminant set).

If $b^{\prime} z_{x}^{\prime}=a^{\prime} z_{y}^{\prime}$ then we have representation (1) outside of the set $\left\{a^{\prime}(x)=b^{\prime}(y)=0\right\}$ (Lemma 1). If $z_{x}^{\prime} \neq 0$ in some point then we can write $b^{\prime}=a^{\prime} z_{y}^{\prime} / z_{x}^{\prime}$. It is clear that the criterion of existence of such $b$ is $\left(a^{\prime} z_{y}^{\prime} / z_{x}^{\prime}\right)_{x}^{\prime}=0$. If $z_{y}^{\prime} a^{\prime} \neq 0$ then we can write this equation as $-a^{\prime \prime} / a^{\prime}=\left(z_{x y}^{\prime \prime} z_{x}^{\prime}-z_{x x}^{\prime \prime} z_{y}^{\prime}\right) /\left(z_{x}^{\prime} z_{y}^{\prime}\right)$. It is clear that the criterion of existence of such $a$ is

$$
\left(\left(z_{x y}^{\prime \prime} z_{x}^{\prime}-z_{x x}^{\prime \prime} z_{y}^{\prime}\right) /\left(z_{x}^{\prime} z_{y}^{\prime}\right)\right)_{y}^{\prime}=0
$$

and then

$$
z_{x}^{\prime} z_{y}^{\prime}\left(z_{x x y}^{\prime \prime \prime} z_{y}^{\prime \prime}-z_{x y y}^{\prime \prime \prime} z_{x}^{\prime}\right)+z_{x y}^{\prime \prime}\left(\left(z_{x}^{\prime}\right)^{2} z_{y y}^{\prime \prime}-\left(z_{y}^{\prime}\right)^{2} z_{x x}^{\prime \prime}\right)=0
$$

We can continue.

\section{Statement 3 .}

(a) Let us assume that $z$ is a holomorphic function in a domain D, it satisfies relation (3) in this domain, and $z_{x}^{\prime} z_{y}^{\prime} \neq 0$ in $p=\left(x_{0}, y_{0}\right) \in D$. Then function $z$ has representation (1) in a neighbourhood of $p$.

(b) This representation has an analytic continuation along all paths in $D \backslash\left\{z_{x}^{\prime} z_{y}^{\prime}=0\right\}$.

(c) If $Z$ is an analytical function generated by $z$ then representation (1) has an analytic continuation along all paths of continuation of $Z$ except for the paths crossing the discriminant set $\left\{z_{x}^{\prime} z_{y}^{\prime}=0\right\}$.

Example 4. Let $z=x y$. It is easy to check that condition (3) is satisfied. We have $-a^{\prime \prime} / a^{\prime}=$ $\left(z_{x y}^{\prime \prime} z_{x}^{\prime}-z_{x x}^{\prime \prime} z_{y}^{\prime}\right) /\left(z_{x}^{\prime} z_{y}^{\prime}\right)=1 / x$. It means that $a(x)=\lambda \ln (x)+\mu$. Then $b^{\prime}=a^{\prime} z_{y}^{\prime} / z_{x}^{\prime}=\lambda / y$ and $b(y)=\lambda \ln (y)+\nu$. We have $x y=c(\lambda(\ln (x y))+\mu+\nu)$. If $T=\lambda(\ln (x y))+\mu+\nu)$, then

$$
c(T)=\exp \left(\frac{T-(\mu+\nu)}{\lambda}\right) .
$$

In this example the domain of analyticity for $z(x, y)$ and the domain of analyticity for representation $S(c, b, a)(x, y)$ do not coincide. Function $z$ is holomorphic everywhere but representation $S(c, b, a)(x, y)$ is analytic only outside of $x y=0$, functions $a$ and $b$ have singular points and they are multivalued functions. In this connection we need a new definition.

\section{Definition 5.}

(a) Function $f$ is analytic almost everywhere function in a domain $D$ if some germ of $f$ has an analytic continuation along all paths in $D$ except for the paths crossing some proper analytic subset of $D$ (the discriminant set).

(b) Let us assume that $f$ is a germ of an analytic function in some point. We say that another germ $g$ in this point has an analytic continuation along almost all paths of the continuation of $f$ if for any element of $f$ in some domain $D$ function $g$ has an analytic continuation along almost all paths in $D$ (in the sense of $(a)$ ). 

in $\mathbf{C}^{2}$.

Now we can say that representation $c(a(x)+b(y))$ of function $x y$ is analytic almost everywhere

Let us consider this result in the context of differential algebra. Let us use the Ritt differential algebra $\mathcal{R}$ over the field of rational numbers. Let $\left(\partial_{x}, \partial_{y}\right)$ be two commuting differentiations in $\mathcal{R}$, and $(c, b, a, z)$ is a set of unknowns. We can write

$$
\text { eqs }=\left\{\partial_{y} a=0, \partial_{x} b=0,\left(\partial_{y} b\right)\left(\partial_{x} c\right)-\left(\partial_{x} a\right)\left(\partial_{y} c\right)=0, c-z=0\right\} .
$$

The first equation means that function $a$ is a function of $x$, the second one means that function $b$ is a function of $y$, and the third one means that function $c$ is a function of $a+b$. We can say that eqs expresses the fact that $z$ has complexity one. This equation generates a radical differential ideal $I$ in $\mathcal{R}$. Using notation given in [2], we can write $I=\{e q s\}$. Let us introduce the monomial order in the following manner $\left(\partial_{y} \succ \partial_{x}\right)$ and $(c \succ b \succ a \succ z)$. This order allows us to exclude some variables from equations. This algorithm was realized above. In the general case such algorithm is based on the Groebner basis method, and its finiteness is guaranteed by the Ritt-Rodenbach theorem ( [1,2], Th. 7.1.).

The representation of functions as composition of complexity more than one can be defined by induction [3]. Namely, a function of complexity $(n+1)$ has the form $z=C\left(A_{n}(x, y)+B_{n}(x, y)\right)$ where $A_{n}$ and $B_{n}$ have complexity $n$. Let us consider a set of $2^{(n+1)}-1$ functions of one variable

$$
\left(A, A_{0}, A_{1}, A_{00}, A_{01}, A_{10}, A_{11}, A_{000}, \ldots, A_{11 \ldots 1}\right) .
$$

Function $A$ is a function of $\left(A_{0}+A_{1}\right)$, function $A_{0}$ is a function of $\left(A_{00}+A_{01}\right)$, function $A_{1}$ is a function of $\left(A_{10}+A_{11}\right)$ and so on until the depth $n$. Functions of $n$-th level have indexes of lengths $n$. Function $A_{j_{1} \ldots j_{n-1} 0}$ depend on $x$ and $A_{j_{1} \ldots j_{n-1} 1}$ depend on $y$ only.

Let us introduce a lexicographical order of unknowns

$$
A \succ A_{0} \succ A_{1} \succ A_{00} \succ \cdots \succ A_{11 \ldots 1} \succ z .
$$

Then we designate these unknowns according to the following order

$$
B_{1}=A, B_{2}=A_{0}, B_{3}=A_{1}, \ldots, A_{11 \ldots 1}=B_{2^{n+1}-1} .
$$

Let $B=\left(B_{1}, \ldots, B_{N}\right)$ be the set of unknowns for the construction of the composition of complexity $n$. We have $N=2^{n+1}-1$.

We describe an algorithm to construct a function of two variables from the set $B$. This algorithm is called full scheme of complexity $n$. We also consider other schemes.

Definition 6. Let B be a finite set of analytical functions of one variable (functional unknowns). Let us assume that there is an algorithm $S$ that allows us to obtain function of two variables $x$, $y$ and $B$ (as data) by substitutions and additions. If each function from set $B$ is used in $S$ only once then we say that $S$ is a scheme of composition.

If instead of functional unknowns $B$ we substitute the set of fixed germs $b$ then we obtain the germ of analytical functions of two variables $z(x, y)=S(b)(x, y)$ or nothing (the domain of definition may be empty).

For example $S_{1}(a, b, c, p, q, r, s)(x, y)=s(c(a(x)+b(y))+r(p(x)+q(y))$ is full scheme of complexity two. If we set $q=0$ then we obtain $S_{2}(a, b, c, r, s)(x, y)=s(c(a(x)+b(y))+r(x))$. This is a non-complete scheme of complexity two. We can write $S_{2} \prec S_{1}$.

Each scheme $S$ corresponds to the class of germs $C l(S)=\{z(x, y)=S(B)(x, y)\}$.

For any scheme $S_{1}$ there is a way to obtain another scheme $S_{2}$. Namely, let us assume that some functions in data $B_{1}$ for $S_{1}$ are zero functions. The remaining part of $B_{1}$ is denoted by $B_{2}$. 
The restriction $S_{1}$ to such data is a new scheme $S_{2}$. In such case we say that $S_{2}$ is less complicated than $S_{1}$ and it is symbolized as $S_{2} \prec S_{1}$. This relation is a partial order on the set of schemes. It is clear that if $S_{2} \prec S_{1}$ then $C l\left(S_{2}\right) \subset C l\left(S_{1}\right)$. Obviously, every class $S$ is a subset of some full class for some complexity $n$. The minimum of such $n$ is called the complexity of $S$.

Definition 7. Let us assume that a germ of analytical function $z(x, y)$ have representation $z(x, y)=S(B)(x, y)$. We say that scheme $S$ is minimal for $z$ if there is no such representation $z(x, y)=\tilde{S}(\tilde{B})(x, y)$ of $z$ with a scheme $\tilde{S} \neq S$ where $\tilde{S} \prec S$.

Let us consider the equation $z=S(B)$. This equation has a lot of differential corollaries. Our goal is the complete elimination of functions of the set $B$ from this equation. First we eliminate function $B_{1}$ then function $B_{2}$ and so on. Lemma 1 allows us to write the criterion of existence of $B_{1}$ as some differential equation on $\left(B_{2}, \ldots, B_{N}, z\right)$. We obtain analyticity of $B_{1}$ almost everywhere in the domain where $\left(B_{2}, \ldots, B_{N}, z\right)$ are analytic. Then we consider to $B_{2}$. Step number $m$ is the elimination of $B_{m}$. We obtain polynomial differential equations $P_{m}\left(B_{m+1}, \ldots, B_{N}, z\right)=0$. In this process the differential order, degrees and the number of equations increase. After the elimination of the last function $B_{N}$ we have a set of polynomial differential equations on $P_{N}(z)=0$ as criterion of existence of representation $z=S(B)$ and the proof of analyticity of this representation. $P_{N}(z)=0$ if and only if $z \in C l(S)$.

One can see that the following statement is correct.

Statement 8. If $z(x, y)=S(B)(x, y), S$ is minimal for $z$, and $P_{m}\left(B_{m+1}, \ldots, B_{N}\right)=0$ is criterion of existence of $B_{m}$ then this system is non-trivial. It means that if $\left(B_{m+1}, \ldots, B_{N}\right)$ is the solution then there is no function from this set without restriction (non arbitrary), and $B_{m}$ is not arbitrary also.

\section{Theorem 9.}

(a) Let us assume that $z(x, y)$ is a germ of holomorphic function at some point $p$, and $z$ has representation with minimal scheme $S$ with the data $B$, i.e. $z(x, y)=S(B)(x, y)$. Then this representation has an analytical continuation along almost all paths of the continuation of $z$.

(b) For any scheme $S$ there exists an algorithm of construction of the differential polynomial system $d(S)(Z)=0$ such that the analytic function $z$ in a domain $D$ has representation with a scheme $S$ almost everywhere in $D$ if and only if $d(S)(Z)=0$. In this case $z$ has some minimal representation with scheme $\tilde{S}$ where $\tilde{S} \prec S$.

(c) The set of functions $B$ for the minimal scheme of representation $z$ is unique up to a finite set of constants.

Proof: Let $\mathcal{R}$ be the differential Ritt algebra with two commuting differentiations $\left(\partial_{x}, \partial_{y}\right)$ over the field of rational numbers. Let us consider a set of ordered unknowns $B=$ $\left(B_{1}, B_{2}, B_{3}, \ldots, B_{N}, z\right)$. One can write the system of differential equations that are equivalent to the representation $z==S(B)(x, y)$. It can be done by Lemma 1 . For example, $B_{1}$ is a function of one variable from $\left(B_{2}+B_{3}\right)$. This is equivalent to the relation

$$
\partial_{y}\left(B_{2}+B_{3}\right) \partial_{x} z-\partial_{x}\left(B_{2}+B_{3}\right) \partial_{y} z=0 .
$$

Similar relation holds for $B_{2}$ and so on. When we write all these equations we obtain the finite system of equations $e q=0$. The set of this differential polynomials generates the radical differential ideal $I(e q)$ in $\mathcal{R}$. This ideal has the finite basis that is consistent with the order of unknowns (Groebner basis). In accordance with the order of unknowns this basis has the form $P, P_{N}, \ldots, P_{1}$. The set of equations $P$ depends only on $z$. The set of equations $P_{j}$ depends on $\left(B_{j}, B_{j+1}, \ldots, B_{N}, z\right)$.

The last function $B_{N}$ depends on one variable (for example $x$ ). Consider the set of equations $P_{N}\left(B_{N}(x), z(x, y)\right)=0$. One can choose some $y=y_{0}$ such that among expressions 
$P_{N}\left(B_{N}(x), z\left(x, y_{0}\right)\right)$ there is one that is not equal to zero identically. We obtain some analytic ordinary equation on $B_{N}(x)$. The solution to this equation is analytic outside the discriminant set (zero set of the separant), and it depends on a finite set of constants. Equations $P(z)=0$ guarantee that this solution will satisfy other equations of the set $P_{N}\left(B_{N}, z\right)=0$. Then we consider the group of equations $P_{N-1}\left(B_{n-1}, B_{N}, z\right)=0$ and we obtain analyticity of $B_{N-1}$ and its dependency on a finite set of constants. This procedure can be continued. For $B_{m}$ we have the system $P_{m}\left(B_{m}, B_{m+1}, \ldots, B_{N}, z\right)=0$. Functions $\left(B_{m+1}, \ldots, B_{N}\right)$ were defined before. These functions are analytic almost everywhere where $z$ is analytic, and they depend on a finite set of constants. Function $B_{m}$ depends on the variable $t$ and $t=B_{j}(x, y)+B_{j+1}(x, y)$ where $j>m$. If we change from variables $(x, y)$ to variables $(x, t)$ then $B_{m}$ is a function of one variable $B_{m}=B_{m}(t)$ and systems

$$
P_{m}\left(B_{m}, B_{m+1}, \ldots\right)=0, \quad P_{m+1}\left(B_{m+1}, \ldots, B_{N}\right)=0, \ldots
$$

are polynomial differential systems in the coordinate system $(x, t)$. We also have analyticity of $B_{m}$ almost everywhere and uniqueness up to a finite set of constants. From $P_{j}=0$ for $j=m+1, \ldots, N$ and $P=0$ we obtain that this $B_{m}$ satisfies all equations from set $P_{m}=0$. On the last step, we eliminate $B_{1}$ and obtain our representation $z=S(B)(x, y)$ as a composition with a scheme $S$. Let $d(S)(z)=P(z)$. Then $d(S)(z)=0$ is the criterion of the existence of a representation with the scheme $S$. If for some $z d(S)(z) \neq 0$ then representation does not exist. If $d(S)(z)=0$ then $z=S(B)(x, y)$ such that $B$ was constructed above. The theorem is proved.

Corollary 10. If a germ $z$ of analytic function $Z$ at some point has a representation with scheme $S$, i.e. $z=S(B)(x, y)$ then it is true for its continuations along almost all paths of continuations for $Z$.

In this sense we can say that a full analytical function $Z$ has a representation with a scheme $S$ and it has a certain complexity $n$.

Each scheme of composition $S$ corresponds to some objects:

- The class $C l(S)$ of analytical functions $Z$ with a representation $Z=S(B)(x, y)$.

- The system of equations $d(S)(z)=0$ that defines the class $C l(S)=\{z: d(S)(z)=0\}$.

- The radical differential ideal $I(S)=\{d(S)\}$ generated by $d(S)$.

This is the list of obvious properties.

\section{Statement 11.}

(a) $Z \in C l(S)$ if and only if $d(S)(z)=0$ for some germ $z$ of function $Z$.

(b) $Z \in C l(S)$ if and only if $d(S)(z)=0$ for any germ $z$ of function $Z$.

(c) $S_{1} \prec S_{2}$ if and only if $C l\left(S_{1}\right) \subset C l\left(S_{2}\right)$.

(d) $S_{1} \prec S_{2}$ if and only if $I\left(S_{1}\right) \supset I\left(S_{2}\right)$.

The research was supported by RFBR grants 17-01-00592 A and 18-51-41011 Uzb t.

\section{References}

[1] J.F.Ritt, Differential algebra, American mathematical society, 1950.

[2] I.Kaplansky, Introduction to differential algebra, Moscow, Izd. Inostr. Lit., 1959.

[3] V.K.Beloshapka, Analytic complexity of functions of two variables, Russian J. Math. Phys., 14(2007), no. 3, 243-249. 


\section{О декомпозиции функций конечной аналитической сложности}

Валерий К. Белошапка

Механико-математический факультет МГУ им. Ломоносова

Воробьевы горы, Москва, 119991

Россия

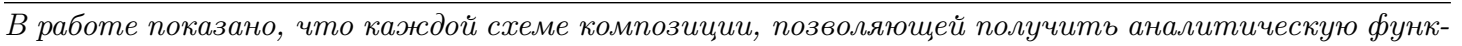
цию двух переменных из аналитических функиий одного переменного и сложения, соответстствует система дифберенциально полиномиальных уравнений с рациональными коэбфициентами такая, что ее аналитические решения и только они разлагаются в композицию с данной схемой (система уравнений схемъ). Это, в частности, доказывает независимость для аналитической функции минимальной схемы композиции от точки и ростка. В работе описан алгоритм получения представления функции в виде композиции с данной схемой и построения системы уравнений схемы.

Ключевые слова: схема декомпозичии, представление функций. 